\title{
Altered peripapillary vessel density and nerve fiber layer thickness in thyroid-associated ophthalmopathy using optical coherence tomography angiography
}

\author{
Huan Jian • Yujiao Wang $\cdot$ Luyu Ou $\cdot$ Weimin He
}

Received: 2 April 2021 / Accepted: 22 September 2021 / Published online: 12 October 2021

(C) The Author(s) 2021

\begin{abstract}
Objective To measure the peripapillary vessel density (VD) and retinal nerve fiber layer thickness (RNFLT) in thyroid-associated ophthalmopathy (TAO) and dysthyroid optic neuropathy (DON) patients using optical coherence tomography angiography (OCTA), and determine their prognostic relevance.

Methods Forty-three TAO patients with or without DON (82 eyes in total) and 26 healthy subjects (52 eyes) were enrolled. All participants underwent ophthalmology and endocrinology tests. The peripapillary VD in retinal peripapillary capillary layer and RNFLT were analyzed using OCTA images. Multiple linear regression analysis was used to assess the relationship between peripapillary VD /RNFLT and the relevant factors.

Results The total peripapillary VD and RNFLT were significantly lower in the DON patients compared to the other groups $(P<0.001,95 \%$ confidence intervals), and each quadrant of VD and RNFLT showed similar results except temporal RNFLT. No significant
\end{abstract}

Huan Jian and Yujiao Wang have contributed equally to this work.

H. Jian · Y. Wang $\cdot$ L. Ou · W. He $(\bowtie)$

Ophthalmic Laboratory, Department of Ophthalmology,

West China Hospital, Sichuan University, No. 37 Guoxue

Street, Chengdu 610041, Sichuan, China

e-mail: hewm888@hotmail.com difference was seen between the RNFLT and VD of active non-DON (ANDON), inactive non-DON (NDON) patients and normal control (NC) group. Multivariable linear regression model showed that high IOP is an independent risk factor for lower peripapillary VD and RNFLT $(\beta=-0.465$, $P<0.001$ and $\beta=-0.343, P=0.002$ respectively).

Conclusion OCTA parameters are suitable indicators for diagnosing DON. TAO patients with high IOP should be considered at high risk of retinal vessel and nerve fiber layer deterioration. In addition, patients with TAO should be advised to quit smoking since it could affect peripapillary VD and RNFLT.

Keywords Thyroid-associated ophthalmopathy . Peripapillary vessel density · Retinal nerve fiber layer thickness - OCTA · Dysthyroid optic neuropathy

\section{Introduction}

Thyroid-associated ophthalmopathy (TAO) is an autoimmune disease that affects visual acuity, facial appearance and the quality of life. It is prevalent across all age groups, and the most severe cases are usually observed among older males [1]. The exact pathological basis of TAO is unclear at present, although immunological, genetic and environmental factors have been implicated in its onset and development [2]. Given its complex clinical features, a single diagnostic 
method cannot accurately evaluate disease severity. In addition, the current grading and staging systems of TAO, such as NOSPECS [3], CAS [4], VISA system [5] and EUGOGO system [6], are mostly subjective and difficult to follow in clinical practice. The onset of dysthyroid optic neuropathy (DON), a severe complication of TAO caused by optic nerve lesion subsequent to the increased orbital or intraocular pressure, is diagnosed on the basis of vision acuity damage, optic disk swelling or optic nerve compression by CT and MRI [7-9].

The current consensus is to improve the quantitative criteria for grading/staging TAO and diagnosing DON. Optical coherence tomography angiography (OCTA) is a non-invasive technique that can detect blood vessels of the retina and choroid through highresolution and three-dimensional images [10], and measure vessel density (VD). OCTA images have shown that the retinal VD correlates negatively with the clinical activity score (CAS) but has no significant correlation with the NOSPECS classification [11, 12]. Studies show that compared to healthy individuals, TAO patients have lower retinal nerve fiber layer thickness (RNFLT) [13] and higher superficial VD in the macula area $^{10}$. In addition, decreased peripapillary VD correlates with aggravated DON [10, 14]. However, another study did not find any significant difference between the macular nerve fiber layer thickness of TAO patients and normal controls [15]. The aim of our study was to determine the diagnostic utility of OCTA parameters for TAO and DON.

\section{Materials and methods}

Study design and subjects

Forty-three TAO patients (4 unilateral and 39 bilateral; 82 affected eyes in total, male-to-female ratio was $1: 1.48)$ were prospectively enrolled at the West China Hospital from November 2018 to March 2019 based on clinical, imaging and laboratory findings. TAO was diagnosed on the basis of Bartley's criteria [16]: (1) patients with eyelid retraction presenting thyroid disorder, proptosis (more than $20 \mathrm{~mm}$ ), optic nerve dysfunction of unknown cause, enlarged extraocular muscles that limit eye movement, or any combination of the above; (2) patients without lid retraction presenting thyroid dysfunction and proptosis, extraocular muscle involvement or optic nerve dysfunction. Based on the CAS at first examination, the TAO patients were further classified into active DON (ADON) or active non-DON (ANDON) group (CAS $\geq 3$ ) and inactive NDON (NDON) group $(\mathrm{CAS}<3)$ [6]. Subjects with other diseases that result in high intraocular pressure (IOP), ocular trauma or previous surgery, systemic metabolic or vascular disease (such as diabetes or hypertension), poor thyroid control, other systemic connective tissue disease or autoimmune disease, or previous TAO treatment with steroids or radiation were excluded. In addition, 26 healthy subjects ( 52 pairs of eyes, aged 19 to 59 years old, male to female ratio was 1: 1.6) were enrolled as the normal control (NC) group. The inclusion criterion was healthy individuals with normal vision acuity, while the individuals with any kind of ocular disease, high IOP, ocular trauma, previous eye surgery, thyroid disease, or systemic disease (such as metabolic, vascular, connective tissue or autoimmune disease) were excluded.

\section{Ocular assessment}

Patients underwent regular ophthalmology tests, including best corrected visual acuity, IOP, exophthalmos, appearance, eye movement, slit lamp microscopy, fundus examination, perimetry, visual evoked potential examination, and enhancement scanning MRI of the ocular region. Best corrected visual acuity was tested using international standard logarithmic visual acuity chart. IOP was measured three times for each eye using TX-20 noncontact tonometer (Canon Corporation, Japan), and the average was calculated. Proptosis was measured using Hertel exophthalmometer.

Optical coherence tomography angiography

(OCTA) measurement

OCTA images were obtained using Optovue AngioVue $^{\mathrm{TM}}$ (RTVue XR Avanti, Optovue Inc. Fremont, CA. USA) and analyzed with AngioAnalytics 2.0 quantization software, which uses the Split-Spectrum Amplitude Decorrelation Angiography algorithm. The wavelength was $840 \mathrm{~nm}$, scanning frequency was $70,000 \mathrm{~Hz}$, distinguishability of lateral and axial direction were $15 \mu \mathrm{m}$ and $5 \mu \mathrm{m}$ respectively, scanning depth was 2-3 mm, A-scan count was $304 \times 304$, and 
B-scan was repeated twice at the same spot. Motion Correction Technic and DualTrac were applied during the entire procedure. HD Angio Disc $4.5 \mathrm{~mm}$ mode was employed to scan a $4.5 \times 4.5 \mathrm{~mm}$ area surrounding the optic nerve, and the retinal peripapillary capillary (ILM-NFL) layer was the default quantified vessel layer. Each position was measured twice, and peripapillary RNFLT and VD were recorded. All measurements were taken by one ophthalmologist.

\section{Statistical analysis}

The data were analyzed using SPSS 23.0 software (SPSS Inc., Chicago, IL, USA IBM). Qualitative data were calculated as percentages and compared using Chi-square test or Fisher exact test. Quantitative data were calculated as mean \pm standard deviation or medians and quartiles. One-way ANOVA with Bonferroni test was used to compare peripapillary RNFLT and nasal VD across multiple groups. Welch's ANOVA with Games-Howell test was used for age, IOP, peripapillary VD and temporal RNFLT. Kruskal-Wallis test was used for duration and number of affected muscles. Factors significantly correlated with the peripapillary VD or RNFLT were identified by Spearman test or point-biserial correlation and incorporated into the multivariable linear regression model. $\mathrm{P}$ values $<0.05$ were considered statistically significant.

\section{Results}

Baseline data and IOP

Eighty-two eyes of 43 TAO patients and 52 eyes of 26 control subjects were analyzed. The male-to-female ratio was 1:1.6 and 1:1.53, respectively in the NC and TAO groups, and the respective mean ages were $39.88 \pm 12.17$ (range 19-59) and $43.39 \pm 11.09$ (range 22 to 62) years. In the TAO group, 39 subjects $(90.70 \%)$ were affected bilaterally, while $4(9.30 \%)$ had unilateral condition. Furthermore, ADON was diagnosed for 6 eyes, whereas 37 eyes were NDON and 39 eyes were ANDON. Age and gender distribution was significantly different across the NC, ADON, ANDON and NDON groups $(p=0.007,0.019$, respectively). The median duration of ocular signs and TAO symptoms was 7.0 months (2-12 months, ranging from 1 to 120 months). In addition, 36 TAO patients $(83.72 \%)$ had hyperthyroidism with median duration of 8.5 months (4.25-24.00 months, ranging from 1 to 120 months), and 10 (76.92\%) of 13 patients undergoing I ${ }^{131}$ treatment had progressed to hypothyroidism, of which $7(53.85 \%)$ presented aggravated eye symptoms. Twelve of 43 patients were smokers (all males), of which 10 (83.33\%) had smoked for more than 10 years. The IOP was significantly higher in the DON patients compared to the other groups $(p<0.001)$ (Fig. 1). The data are summarized in Table 1. MRI pictures of four groups are presented in Fig. 2.

\section{Peripapillary VD and RNFLT}

The OCTA images of all four groups were analyzed to determine peripapillary VD and RNFLT (Fig. 3, Tables 2 and 3). Total peripapillary VD of the DON group was only $32.18 \pm 5.48 \%$ compared to $52.69 \pm 2.48 \%, 53.31 \pm 2.93 \%$ and $54.26 \pm 2.30 \%$ recorded for the NDON, ANDON and NC groups, respectively ( $P<0.001$ for each pair comparison). Total peripapillary RNFLT in the DON group was $63.47 \pm 15.81 \mu \mathrm{m}$, and was significantly lower compared to that in other groups $(118.68 \pm 11.08 \mu \mathrm{m}$, $122.79 \pm 15.33 \mu \mathrm{m}$ and $118.01 \pm 13.07 \mu \mathrm{m}$ for NDON, ANDON and NC groups respectively; $P<0.001$ for each). Likewise, the superior, inferior and nasal sector peripapillary RNFLT in the DON patients showed significant differences with the corresponding values in the other groups $(p<0.001)$. However, total and regional RNFLT were similar among the NDON, ANDON, and NC groups. The OCTA images of different groups are shown in Fig. 4.

Factors associated with peripapillary VD and RNFLT

Single factor correlation analysis was conducted with peripapillary VD or RNFLT as the dependent variable, and gender, age, duration of TAO, smoking history, thyroid condition, proptosis degree, IOP, number of affected muscles as the independent variables. IOP, gender, thyroid condition and smoking history were significantly correlated to peripapillary $\mathrm{VD}$ and RNFLT, whereas proptosis degree was associated with peripapillary RNFLT $(P<0.05)$ (Table 4$)$. Multivariable linear regression model further identified 


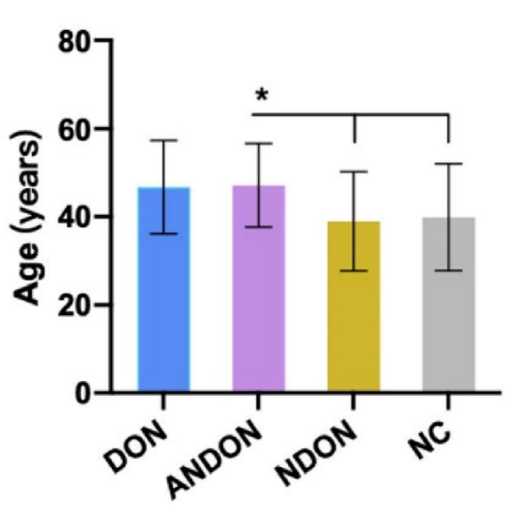

A

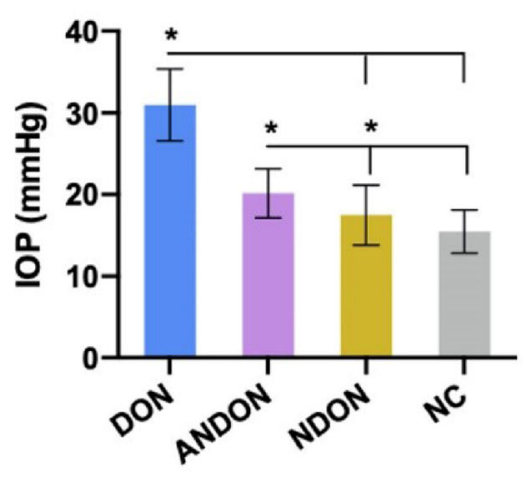

B

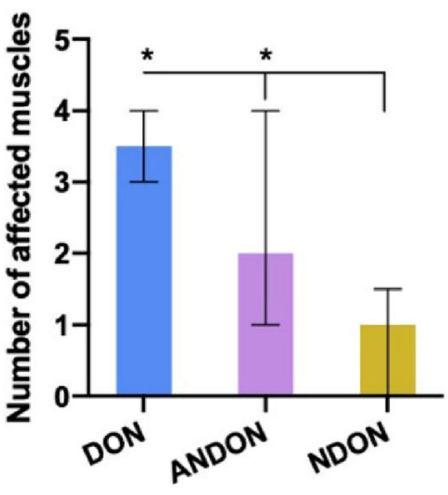

C

Fig. 1 Evaluation of basic information including age $\mathbf{a}$, IOP $\mathbf{b}$, number of affected muscles $\mathbf{c}$ of the four groups. (* $P<0.05$ between indicated groups)

Table 1 Baseline data of all four groups

\begin{tabular}{|c|c|c|c|c|c|c|}
\hline & & DON group & NDON group & ANDON group & NC group & $P$ value \\
\hline$n$ & & 6 & 37 & 39 & 52 & - \\
\hline Male ratio $(\%)$ & & 100 & 37.84 & 33.33 & 38.46 & $0.019^{\mathrm{b}}$ \\
\hline Age (year) & & $46.67 \pm 10.60$ & $38.92 \pm 11.31$ & $47.13 \pm 9.53$ & $39.88 \pm 12.17$ & $0.007^{\mathrm{a}}$ \\
\hline IOP (mmHg) & & $30.98 \pm 8.18$ & $17.48 \pm 3.67$ & $20.16 \pm 3.00$ & $15.44 \pm 2.62$ & $<0.001^{\mathrm{a}}$ \\
\hline Duration (month) & & $\begin{array}{l}21.00 \\
\quad(7.00-24.00)\end{array}$ & $\begin{array}{l}9.00 \\
\quad(2.00-36.00)\end{array}$ & $\begin{array}{l}6.00 \\
\quad(3.00-12.00)\end{array}$ & N/A & $0.08^{\mathrm{c}}$ \\
\hline \multirow[t]{3}{*}{ Thyroid function (\%) } & hyperthyroidism & $6(100 \%)$ & $20(54.05 \%)$ & $26(66.67 \%)$ & N/A & $0.085^{\mathrm{b}}$ \\
\hline & hypothyroidism & $(0.00 \%)$ & $10(27.03 \%)$ & $8(20.51 \%)$ & N/A & $0.385^{\mathrm{b}}$ \\
\hline & $\begin{array}{l}\text { normal thyroid } \\
\text { function }\end{array}$ & $(0.00 \%)$ & $7(18.92 \%)$ & $5(12.82 \%)$ & N/A & $0.554^{\mathrm{b}}$ \\
\hline $\begin{array}{l}\text { Number of affected } \\
\text { muscles }\end{array}$ & & $3.5(3-4)$ & $1(0-1.5)$ & $2(1-4)$ & N/A & $<0.001^{\mathrm{c}}$ \\
\hline
\end{tabular}

N/A: not applicable for this group

${ }^{a}$ Welch's ANOVA

${ }^{\mathrm{b}}$ Fisher exact test

${ }^{\mathrm{c}}$ Kruskal-Wallis test

${ }^{\mathrm{d}}$ ANOVA; $P<0.05$, there is significant difference among groups

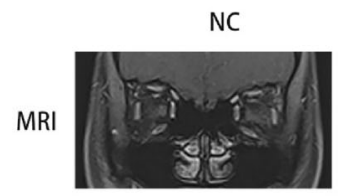

A inactive NDON

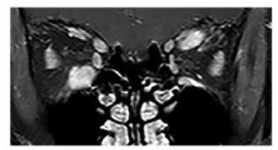

B

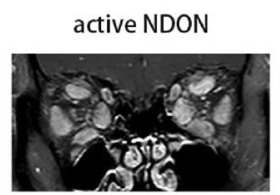

C

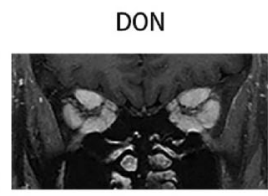

D
Fig. 2 Coronal MRI pictures of the four groups. NC group showed no abnormal muscles. a NDON group. b and ANDON group. c showed varying degrees of enlarged extraocular muscles. DON group showed enlarged extraocular muscles and crowded orbital apex (d) 
Fig. 3 Evaluation of peripapillary VD a and RNFLT $\mathbf{b}$ in the four groups. (* $P<0.05$ between indicated groups)

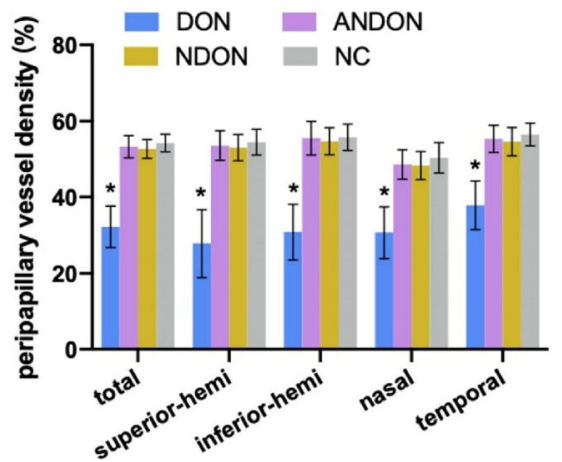

A

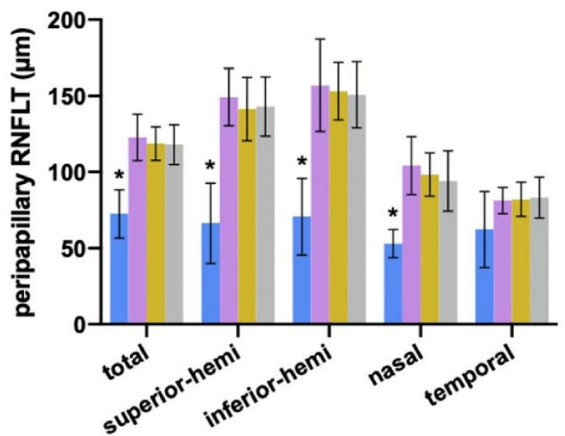

B

Table 2 Peripapillary VD of different groups

\begin{tabular}{llllll}
\hline & DON group & NDON group & ANDON group & NC group & $P$ value \\
\hline Total peripapillary VD (\%) & $32.18 \pm 5.48$ & $52.69 \pm 2.48$ & $53.31 \pm 2.93$ & $54.26 \pm 2.30$ & $<0.001^{\mathrm{b}}$ \\
Superior VD (\%) & $27.80 \pm 8.93$ & $53.03 \pm 3.49$ & $53.68 \pm 3.89$ & $54.50 \pm 3.37$ & $<0.001^{\mathrm{b}}$ \\
Inferior VD (\%) & $30.83 \pm 7.25$ & $54.73 \pm 3.56$ & $55.54 \pm 4.43$ & $55.77 \pm 3.48$ & $<0.001^{\mathrm{b}}$ \\
Nasal VD (\%) & $30.67 \pm 6.74$ & $48.35 \pm 3.69$ & $48.64 \pm 3.85$ & $50.35 \pm 4.00$ & $<0.001^{\mathrm{a}}$ \\
Temporal VD (\%) & $37.83 \pm 6.37$ & $54.65 \pm 3.72$ & $55.36 \pm 3.57$ & $56.49 \pm 2.98$ & $<0.001^{\mathrm{b}}$ \\
\hline
\end{tabular}

${ }^{\mathrm{a} A N O V A}$

belch's ANOVA; $P<0.05$, there is significant difference among the groups

Table 3 Peripapillary RNFLT of different groups

\begin{tabular}{llcccc}
\hline & DON group & NDON group & ANDON group & NC group & $P$ value \\
\hline Total peripapillary RNFLT $(\mu \mathrm{m})$ & $63.47 \pm 15.81$ & $118.68 \pm 11.08$ & $122.79 \pm 15.33$ & $118.01 \pm 13.07$ & $<0.001^{\mathrm{a}}$ \\
Superior RNFLT $(\mu \mathrm{m})$ & $66.40 \pm 26.29$ & $141.41 \pm 20.90$ & $149.29 \pm 18.86$ & $143.06 \pm 19.51$ & $<0.001^{\mathrm{a}}$ \\
Inferior RNFLT $(\mu \mathrm{m})$ & $70.67 \pm 25.05$ & $153.08 \pm 18.88$ & $156.90 \pm 30.36$ & $150.73 \pm 21.65$ & $<0.001^{\mathrm{a}}$ \\
Nasal RNFLT $(\mu \mathrm{m})$ & $53.00 \pm 9.06$ & $98.30 \pm 14.25$ & $104.18 \pm 19.04$ & $94.10 \pm 19.89$ & $<0.001^{\mathrm{a}}$ \\
Temporal RNFLT $(\mu \mathrm{m})$ & $62.17 \pm 24.86$ & $81.95 \pm 11.27$ & $81.18 \pm 8.62$ & $83.10 \pm 13.54$ & $0.274^{\mathrm{b}}$ \\
\hline
\end{tabular}

${ }^{\mathrm{a}} \mathrm{ANOVA}$

belch's ANOVA; $P<0.05$, there is significant difference among the groups

IOP as the only independent factor of peripapillary VD and RNFLT $(\beta=-0.465, \quad P<0.001$, adjusted $R^{2}=0.381$ and $\beta=-0.343, P=0.002$, adjusted $R^{2}=0.234$ respectively; Table 5).

\section{Discussion}

DON is often characterized by irreversible orbital changes, such as extraocular muscles enlargement, fibrosis and fatty degeneration, which significantly increase the orbital volume and restrict the exophthalmos process. This eventually increases the IOP, resulting in compression of the orbital blood vessel and optic nerve [17-19]. Consistent with previous reports, the IOP of DON patients were significantly higher and peripapillary VD was significantly lower compared to that of the other groups. Multivariable linear regression model further confirmed that increased IOP is an independent factor of low 
Fig. 4 OCTA images of the four groups. Peripapillary VD and RNFLT of NC group were normal. NDON group showed minor decrease of peripapillary VD and RNFLT. ANDON and DON groups showed obviously diminished peripapillary VD

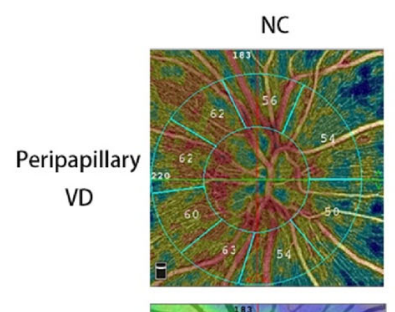

inactive NDON
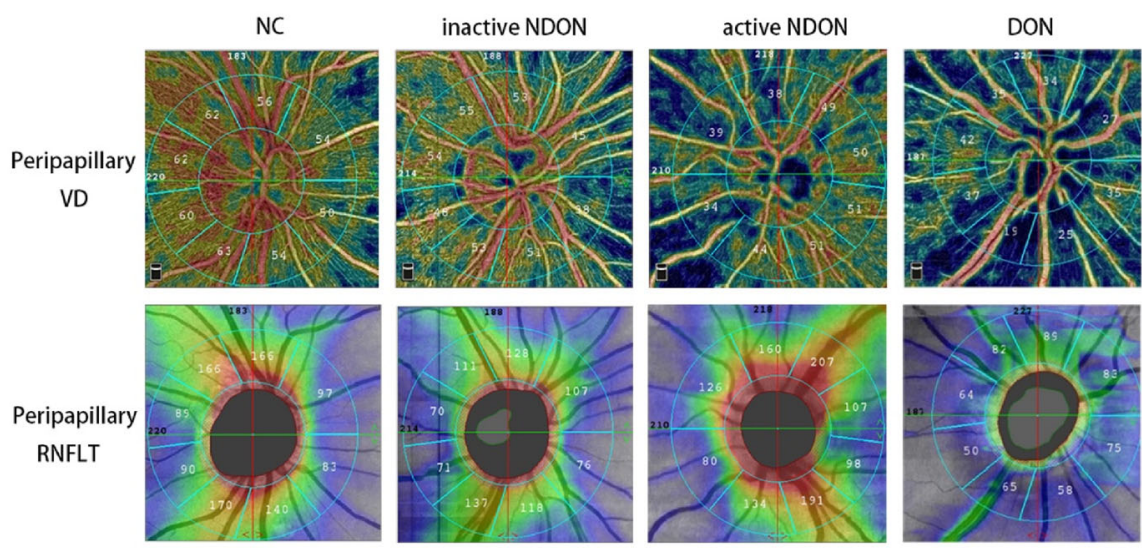

Table 4 Single factor correlation analysis of peripapillary VD and RNFLT

${ }^{\text {a }}$ Spearman test for correlation; ${ }^{b}$ point-biserial correlation

\begin{tabular}{llllll}
\hline & \multicolumn{2}{l}{ VD } & & \multicolumn{2}{l}{ RNFLT } \\
\cline { 2 - 3 } & Correlation Coefficient & $P$ Value & & Correlation coefficient & $P$ Value \\
\hline IOP (mmHg) & -0.277 & $0.012^{\mathrm{a}}$ & & -0.244 & $0.027^{\mathrm{a}}$ \\
Gender & -0.395 & $<0.001^{\mathrm{b}}$ & -0.364 & $0.001^{\mathrm{b}}$ \\
Smoking history & -0.463 & $<0.001^{\mathrm{b}}$ & -0.362 & $0.001^{\mathrm{b}}$ \\
Thyroid condition & -0.231 & $0.037^{\mathrm{b}}$ & -0.231 & $0.037^{\mathrm{b}}$ \\
Proptosis degree $(\mathrm{mm})$ & -0.065 & $0.559^{\mathrm{a}}$ & -0.297 & $0.007^{\mathrm{a}}$ \\
\hline
\end{tabular}

Table 5 Multivariable linear regression analysis of peripapillary VD and RNFLT

\begin{tabular}{|c|c|c|c|c|c|c|c|c|}
\hline & \multicolumn{4}{|l|}{ VD } & \multicolumn{4}{|l|}{ RNFLT } \\
\hline & $\beta$ & $t$ & $P$ & Adjusted R Square & $\beta$ & $t$ & $P$ & Adjusted R Square \\
\hline IOP (mmHg) & -0.465 & -4.822 & $<0.001$ & 0.381 & -0.343 & -3.198 & 0.002 & 0.234 \\
\hline Gender & -0.194 & -1.328 & 0.188 & & -0.269 & -1.555 & 0.124 & \\
\hline Smoking history & -0.12 & -0.768 & 0.445 & & 0.054 & 0.309 & 0.758 & \\
\hline Thyroid condition & -0.063 & -0.667 & 0.507 & & -0.12 & -1.115 & 0.269 & \\
\hline Proptosis degree $(\mathrm{mm})$ & - & - & - & & -0.108 & -0.946 & 0.347 & \\
\hline
\end{tabular}

peripapillary VD. Another study also reported a similar relationship between IOP and optic nerve head perfusion [18]. While pressure from enlarged extraocular muscles and orbital fat compress the eyeball, increased episcleral vein pressure caused by high orbital tension can lead to higher outflow resistance of aqueous humor, resulting in high IOP [19]. Therefore, the decrease in peripapillary VD might be the result of orbital blood vessel compression and increased IOP. The total peripapillary RNFLT was also markedly lower in the DON patients, and affected by the IOP. It is possible that hypoxic-ischemia resulting from increased IOP and the concomitant decrease in blood supply leads to the loss of retinal nerve cells. In addition, the RNFLT in the superior, inferior and nasal peripapillary areas were lower in the DON group, which might due to increased IOP in these susceptible areas.

Smoking was significantly correlated with peripapillary VD and RNFLT in our cohort, and TAO patients with a history of smoking had thinner RNFL and fewer retinal blood vessels compared to the non-smokers [20]. Previous studies have also established smoking as a risk factor for TAO progression $[6,21]$. There is 
also evidence that peripapillary VD in active TAO is lower than that during inactive disease [15], although Lei Ye et all [14] reported increased macular VD in TAO patients. Furthermore, Kyle T Lewis et all [10] found that peripapillary VD decreased in DON patients after decompression surgery and improved the symptoms, whereas one patient showed deterioration of symptoms with increased peripapillary VD. However, we did not detect any significant difference between the peripapillary VD of active and inactive TAO patients, which could be attributed to the small sample size. Since peripapillary VD in the TAO eyes is easily affected by orbital and intraocular pressure and appears earlier than visual impairment [22], it is a reliable predictive indicator of DON and visual prognosis. However, differences in sample size, racial diversity, parameter settings of OCTA and inclusion criteria for TAO patients may have resulted in the discrepancies among previous studies. Future investigation is needed to validate the predictive and diagnostic potential of OCTA in TAO and DON.

There are several limitations in our study that ought to be considered. First, selection bias could not be ruled out since all recruited patients visited the hospital for ocular symptoms. In addition, we did not have the long-term follow-up data to evaluate the true predictive value of reduced peripapillary VD in TAO. Second, the sample size was relatively small, which limits the generalizability of the results. Furthermore, ocular blood supply is affected by multiple factors such as hypertension, and variations in VD across the different retinal areas may also have an effect on OCTA results. Therefore, these factors should be taken into consideration when analyzing the activity and severity of TAO by OCTA.

In conclusion, reduced peripapillary VD as measured by OCTA is a promising prognostic indicator of optic neuropathy in TAO and can diagnose early subclinical DON. High IOP should be considered a risk factor for decreased retinal vessel and nerve fiber layer thickness in TAO patients. Further studies should be conducted on larger cohorts to validate the present findings.

Author contributions H.J. and Y.W. analyzed the medical data of all subjects and drafted the manuscript, so they contributed equally to this study. L.O. was responsible for collecting medical data and preliminary organized these data. W.H., as the corresponding author, ensured the data were conducted appropriately and the result and discussion were accurately presented in our manuscript.

Funding This study was supported by a grant from the 1.3.5 Project for Disciplines of Excellence-Clinical Research Incubation Project, West China Hospital, Sichuan University (2018HXFH024).

Data availability The datasets used or analyzed during the current study are available from the corresponding author on reasonable request.

\section{Declarations}

Conflict of interest The authors have no conflicts of interest to declare.

Ethical approval The study was conducted in accordance with the guidelines of the Declaration of Helsinki, and approved by the review board of West China Hospital, Sichuan University (Chengdu, China). Patients provided written informed consent for data and image publication.

Consent to participate Informed consent was obtained from all individual participants included in the study.

Open Access This article is licensed under a Creative Commons Attribution 4.0 International License, which permits use, sharing, adaptation, distribution and reproduction in any medium or format, as long as you give appropriate credit to the original author(s) and the source, provide a link to the Creative Commons licence, and indicate if changes were made. The images or other third party material in this article are included in the article's Creative Commons licence, unless indicated otherwise in a credit line to the material. If material is not included in the article's Creative Commons licence and your intended use is not permitted by statutory regulation or exceeds the permitted use, you will need to obtain permission directly from the copyright holder. To view a copy of this licence, visit http://creativecommons.org/licenses/by/4.0/.

\section{References}

1. Perros $\mathrm{P}$, Crombie AL, Matthews JN, Kendall-Taylor $\mathrm{P}$ (1993) Age and gender influence the severity of thyroidassociated ophthalmopathy: a study of 101 patients attending a combined thyroid-eye clinic. Clin Endocrinol (Oxf) 38(4):367-372. https://doi.org/10.1111/j.1365-2265.1993. tb00516.x

2. Wang L, Ma JM (2017) Progression of the pathogenesis of thyroid associated ophthalmopathy. [Zhonghua yan ke za zhi] Chin J Ophthalmol 53(6):474-480. https://doi.org/10. 3760/cma.j.issn.0412-4081.2017.06.018

3. Werner SC (1977) Modification of the classification of the eye changes of Graves' disease. Am J Ophthalmol 83(5):725-727. 9394(77)90140-4 
4. Mourits MP, Koornneef L, Wiersinga WM, Prummel MF, Berghout A, van der Gaag R (1989) Clinical criteria for the assessment of disease activity in Graves' ophthalmopathy: a novel approach. Br J Ophthalmol 73(8):639-644. https:// doi.org/10.1136/bjo.73.8.639

5. Dolman PJ, Rootman Jack (2006) VISA Classification for Graves orbitopathy. Ophthalmic Plast Reconstr Surg 22(5):319-324. 0000235499.34867 .85

6. L Bartalena, L Baldeschi, K Boboridis, A Eckstein, GJ Kahaly, C Marcocci, P Perros, M Salvi, WM Wiersinga, Orbitopathy European Group on Graves (2016) The 2016 European thyroid association/European group on Graves' orbitopathy guidelines for the management of Graves' orbitopathy. Eur Thyroid J 5 (1): 9-26.Doi: https://doi.org/ 10.1159/000443828

7. D Mckeag, C Lane, JH Lazarus, L Baldeschi, K Boboridis, AJ Dickinson, AI Hullo, G Kahaly, G Krassas, C Marcocci, M Marinò, MP Mourits, M Nardi, C Neoh, J Orgiazzi, P Perros, A Pinchera, S Pitz, M FPrummel, MS Sartini, WM Wiersinga, Orbitopathy European Group on Graves (2007) Clinical features of dysthyroid optic neuropathy: a European Group on Graves' Orbitopathy (EUGOGO) survey. Br J Ophthalmol 91(4): 455-458.Doi: https://doi.org/10.1136/ bjo.2006.094607

8. Rutkowska-Hinc B, Maj E, Jabłońska A, Milczarek-Banach J, Bednarczuk T, Miśkiewicz P (2018) Prevalence of radiological signs of dysthyroid optic neuropathy in magnetic resonance imaging in patients with active, moderate-tosevere, and very severe graves orbitopathy. Eur Thyroid J 7(2):88-94. https://doi.org/10.1159/000486828

9. Weis E, Heran MKS, Jhamb A, Chan AK, Chiu JP, Hurley MC, Rootman J (2011) Clinical and soft-tissue computed tomographic predictors of dysthyroid optic neuropathy: refinement of the constellation of findings at presentation. Arch Ophthalmol 129(10):1332-1336. https://doi.org/10. 1001/archophthalmol.2011.276

10. Lewis KT, Bullock JR, Drumright RT, Olsen MJ, Penman AD (2019) Changes in peripapillary blood vessel density in Graves' orbitopathy after orbital decompression surgery as measured by optical coherence tomography angiography. Orbit 38(2):87-94. https://doi.org/10.1080/01676830.2018. 1446539

11. Yufei Wu, Yunhai Tu, Bao Lulu, Chaoming Wu, Zheng J, Wang J, Fan Lu, Shen M, Chen Q (2019) Reduced retinal microvascular density related to activity status and serum antibodies in patients with Graves' ophthalmopathy. Curr Eye Res 45(5):576-584. https://doi.org/10.1080/02713683. 2019.1675177

12. Mihailovic N, Lahme L, Rosenberger F, Hirscheider M, Termühlen J, Heiduschka P, Grenzebach U, Eter N, Alnawaiseh M (2019) Altered retinal perfusion in patients with inactive graves' ophthalmopathy using optical coherence tomography angiography. Endocr Pract 26(3):312-317. https://doi.org/10.4158/EP-2019-0328

13. Forte R, Bonavolontà P, Vassallo P (2010) Evaluation of retinal nerve fiber layer with optic nerve tracking optical coherence tomography in thyroid-associated orbitopathy. Ophthalmologica 224(2):116-121. https://doi.org/10.1159/ 000235925

14. Ye L, Zhou S-S, Yang W-L, Bao J, Jiang N, Min Y-L, Yuan Q, Tan G, Shen M, Shao Y (2018) Retinal microvasculature alteration in active thyroid-associated ophthalmopathy. Endocr Pract 24(7):658-667. https://doi.org/10.4158/EP2017-0229

15. Tehrani MJ, Mahdizad Z, Kasaei A, Fard MA (2019) Early macular and peripapillary vasculature dropout in active thyroid eye disease. Graefes Arch Clin Exp Ophthalmol 257(11):2533-2540. https://doi.org/10.1007/s00417-01904442-8

16. Bartley GB, Gorman CA (1995) Diagnostic criteria for Graves' ophthalmopathy. Am J Ophthalmol 119(6):792-795. https://doi.org/10.1016/s00029394(14)72787-4

17. Konuk O, Onaran Z, Oktar SO, Yucel C, Unal M (2009) Intraocular pressure and superior ophthalmic vein blood flow velocity in Graves' orbitopathy: relation with the clinical features. Graefes Arch Clin Exp Ophthalmol 247(11):1555-1559. https://doi.org/10.1007/s00417-0091144-0

18. Jia Y, Morrison JC, Jason Tokayer O, Tan LL, Baumann B, Lu CD, Choi W, Fujimoto JG, Huang D (2012) Quantitative OCT angiography of optic nerve head blood flow. Biomed Opt Express 3(12):3127-3137. https://doi.org/10.1364/ BOE.3.003127

19. Thyparampil P, Yen MT (2016) Compressive optic neuropathy in thyroid eye disease. Int Ophthalmol Clin 56(1):51-67. 0000000000000096

20. Moschos MM, Nitoda E, Laios K, Ladas DS, Chatziralli IP (2016) The impact of chronic tobacco smoking on retinal and choroidal thickness in greek population. Oxid Med Cell Longev 2016:2905789. https://doi.org/10.1155/2016/ 2905789

21. Wang YJ, He WM (2013) Clinical analysis of 403 cases of thyroid associated ophthalmopathy. [Zhonghua yan ke za zhi] Chin J Ophthalmol 49(8):685-90

22. Zhang T, Xiao W, Ye H, Chen R, Mao Y, Yang H (2019) Peripapillary and Macular vessel density in dysthyroid optic neuropathy: an optical coherence tomography angiography study. Invest Ophthalmol Vis Sci 60(6):1863-1869. https:// doi.org/10.1167/iovs.18-25941

Publisher's Note Springer Nature remains neutral with regard to jurisdictional claims in published maps and institutional affiliations. 